\title{
Estimates of the statistical two-dimensional spatial structure in rain over a small network of disdrometers
}

\author{
A. R. Jameson ${ }^{1,3}$ - M. L. Larsen ${ }^{2}$
}

Received: 30 May 2015/ Accepted: 26 January 2016/Published online: 10 February 2016

(C) The Author(s) 2016. This article is published with open access at Springerlink.com

\begin{abstract}
Microphysical understanding of the variability in rain requires a statistical characterization of different drop sizes both in time and in all dimensions of space. Temporally, there have been several statistical characterizations of raindrop counts. However, temporal and spatial structures are neither equivalent nor readily translatable. While there are recent reports of the one-dimensional spatial correlation functions in rain, they can only be assumed to represent the two-dimensional (2D) correlation function under the assumption of spatial isotropy. To date, however, there are no actual observations of the (2D) spatial correlation function in rain over areas. Two reasons for this deficiency are the fiscal and the physical impossibilities of assembling a dense network of instruments over even hundreds of meters much less over kilometers. Consequently, all measurements over areas will necessarily be sparsely sampled. A dense network of data must then be estimated using interpolations from the available observations. In this work, a network of 19 optical disdrometers over a $100 \mathrm{~m}$ by $71 \mathrm{~m}$ area yield observations of drop spectra every minute. These are then interpolated to a $1 \mathrm{~m}$ resolution grid. Fourier techniques then yield estimates of the 2D spatial correlation functions. Preliminary examples using this technique found that steadier, light rain decorrelates spatially faster than does the convective rain, but in
\end{abstract}

Responsible Editor: C. Simmer.

$\triangle$ A. R. Jameson

arjatrjhsci@verizon.net

1 RJH Scientific, Inc., El Cajon, CA, USA

2 Department of Astronomy and Physics, College of Charleston, Charleston, SC, USA

35625 N. 32nd St., Arlington, VA 22207-1560, USA both cases the 2D spatial correlation functions are anisotropic, reflecting an asymmetry in the physical processes influencing the rain reaching the ground not accounted for in numerical microphysical models.

\section{Introduction}

The existence of temporal and spatial structures in rain is widely recognized. One of the primary motivations for studies of this structure has long been hydrological applications, specifically flood predictions and flood warnings. However, a continuing dilemma facing these applications is the general sparseness of instruments for directly measuring the rain (e.g., rain gages). The discovery in the 1940s that radars detect rain opened the possibility of using remote sensing to 'fill-in' for missing rain gage measurements. The quest to make such radar measurements sufficiently quantitative led to detailed studies of the microstructure of the rain starting with the studies of raindrop size distributions of Wexler (1948), Marshall and Palmer (1948), and Best (1950) which are still continuing today (Uijlenhoet et al. 2003a, b; Steiner et al. 2004).

Unfortunately, the dimensions of the radar and satellite beams are often from one up to several kilometers. This is known to affect retrievals using power laws between the radar reflectivity factor and rainfall rate (Sassi et al. 2014), for example. Hydrological applications and flood warnings, however, sometimes require finer resolution usually attempted through downscaling of radar measurements (e.g., Lanza et al. 2001) and outputs from numerical models (e.g., Venugopal et al. 1999a, b; Droegemeier et al. 2000; Ferraris et al. 2002; Rebora et al. 2006; Huebener et al. 2007). 
Conversely, observations of rainfall parameters are sometimes used as inputs into numerical forecast models in an attempt to 'adjust' the computations, to improve forecasts and to evaluate the quality of these forecasts (e.g., Tustison et al. 2001; Ahrens and Beck 2008). Often these models, designed for much larger scales, even use input from 8 inch diameter rain gage measurements which must then be 'upscaled' to match the kilometer grid spacing of the models.

There are other reasons for understanding of the spatial structure of rain itself. These include predictions of communication fading (Crane 1990; Hodges et al. 2005); the translation of space observations of precipitation down to regional scales (e.g., Verlinde 2011); and downscaling of global climate model predictions to regional scales (e.g., Mannshardt-Shamseldin et al. 2010).

For these reasons, research into the variability of rain has generally fallen into two classes of studies, namely, those which characterize the microstructure of the rain and those more focused on what may be called the macrostructure of the rain. The latter is largely focused on the spatial distributions of rainfall rates on scales larger than $1 \mathrm{~km}$ and/or over long times (Lee and Zawadzki 2005; Lee et al. 2009; Tokay and Bashor 2010; Tapiador et al. 2010; Jaffrain and Berne 2012a, b).

It is only recently that it has become possible to begin studying what may be called the macroscale variability of the microstructure of rain in large part with the advent of inexpensive yet reliable disdrometers. The ground-breaking work of Tapiador et al. (2010) and of Jaffrain and Berne (2012a) used observations of spatially separated optical disdrometers to compute the spatial correlations of moments of the drop size distributions (i.e., the rainfall rate, the radar reflectivity factor, mean drop size) between pairs of instruments separated by distances varying from hundreds of meters to a few kilometers as has Jameson et al. (2015a) on scales less than $100 \mathrm{~m}$. As important as these studies are, however, they are only one-dimensional pair correlations, not the complete two-dimensional correlation functions over an area. Yet such two-dimensional information is not only important to science for developing a better understanding of rain formation and for a more complete understanding the origin of coherent radar backscatter from precipitation (Jameson and Kostinski 2010a, b), but also very important for understanding the physics of soil erosion (Kinnell 2005; Caracciolo et al. 2012), a multibillion dollar a year loss to agriculture (Uri and Lewis 1999).

At larger scales, the two dimensionality of rain structures produce the variability that can affect urban flooding, localized flood warnings and both urban (Sangati and Borga 2009) and mountain (Meusburger et al. 2012) runoff planning. Yet all that is known about this structure is what few 1D observations exist in clouds (Kostinski and Jameson 2000; Shaw et al. 2002) and rain (Kostinski et al. 2006; Jameson et al. 2015a). But the processes of precipitation formation are not only variable in time and the vertical direction, as replicated in many numerical simulations; they are also variable in the horizontal plane. That is, one cannot understand all the processes affecting rain formation and evolution by just looking at a point in space or by following a drop in time. For example, recent studies of the evolution of cloud droplets into larger drops emphasize the role played by multi-dimensional droplet clustering (Shaw et al. 1998, 2002; Kostinski and Shaw 2001, 2005). The same likely applies to rain as well.

Because rainfall parameters are random variables, it is necessary to describe any structures statistically. Most of the time, such descriptions are based upon single instruments measuring a time series of drop counts (disdrometers) or upon rainfall accumulations (rain gages) over detector areas of $50-100 \mathrm{~cm}^{2}$, in the case of disdrometers, and typically a few hundred $\mathrm{cm}^{2}$ for rain gages. For both disdrometers and rain gages, most observations are usually made at a nominal temporal resolution of $1 \mathrm{~min}$, but some disdrometers are capable of sub-second resolution. Whatever the temporal resolution, a common statistical tool for studying the structure of rain in time using a disdrometer is the correlation function of counts of different sizes of drops (Kostinski and Jameson 1997; Jameson and Kostinski 2000). As useful as such studies have been, they are not equivalent to one-dimensional (1D) spatial observations (Jameson et al. 2015a). Only recently have there been a few estimates of the 1D spatial correlation functions in rain on the scale of meters (Jameson et al. 2015a) for rain drops and up to $1 \mathrm{~km}$ (Jaffrain and Berne 2012a) and longer (van de Beek et al. 2012) for the rainfall rate and the radar reflectivity factor (Tapiador et al. 2010). At present there are no reports of the two-dimensional (2D) correlation functions over areas of rain using observations.

The reason is the sparseness of instrumentation over even small areas, much less those having kilometer dimensions. As in any field of research, when this occurs, a dense network of measurements over two dimensions must then be estimated using interpolations from the available observations.

Such an approach has obvious pitfalls because it implies a continuous spatial relation among data points when, in fact, none may exist. For example, suppose all of the observations were random numbers. An interpolation scheme would still produce smooth surfaces and non-zero correlation functions. This obvious failure can at least partially be avoided by inspecting the data to be certain that there is continuity in time and space among the observations at the different locations so that any interpolated fields are not artifacts but informed guesses as to what lies 
between the measurements. It must be remembered at all times, however, that the resulting 2D correlation functions only represent estimates of what the actual correlation function may have been, and will underestimate fluctuations in any field with structures both smaller than instrument separation, larger than the size of the array, and due to other factors like field intermittency.

It is also important to use an interpolation method that does not create artificial features that would influence the estimates of the correlation function. In this work, we use commercially available software to implement the conservative interpolation method of Watson (1992) based upon the concept of natural neighbors (Sibson 1981). It is essentially a distance and Voronoi partitions weighted interpolation scheme that conserves the observed observation. Each interpolated field looks much like a sheet of rubber stretched over all of the observation points. Unlike many other interpolation schemes such as those conserving first derivatives, this method does not produce artificial spatial maxima nor minima between observations. This is important because it means that it does not artificially alter the intrinsic correlation function. It is worth dwelling on this a little, since interpolation makes the estimation of the 2D spatial correlation function over areas possible.

First, it is important to note that it is 2D spatial correlation that is responsible for the observed structures in rain. This is demonstrated in Fig. 1 where we begin with a featureless random field of numbers as illustrated in Fig. 1a. Using a one-dimensional decaying exponential function having a correlation length of $X_{1}=15$ units, these random data points can be correlated in two dimensions using the so-called root matrix method described in detail in (Johnson 1994; Jameson and Kostinski 1999; Jameson 2015). The result of this correlation is illustrated in Fig. 1b where there are now structures where none existed previously. While these were just random numbers, the same approach can be used to simulate rain as well (Jameson 2015). Structures are equivalent to the presence of correlation. Hence, if one uses an interpolation method that does not produce structures (as some interpolation methods do) while leaving the observations exactly, it does not, therefore, add spurious correlations. What it does do, however, is yield a smooth, continuous function everywhere except at the point of observations which are exactly matched at each data source. As will be shown below, this allows one to estimate the 2D spatial correlation function over areas. An example of this interpolation will be presented in a subsequent section.

First, however, in the next section, it is necessary to present the quantities being estimated and the methodology for doing so. Subsequent sections will briefly describe the network of instruments, a description of the data being analyzed and an example of the natural neighbor
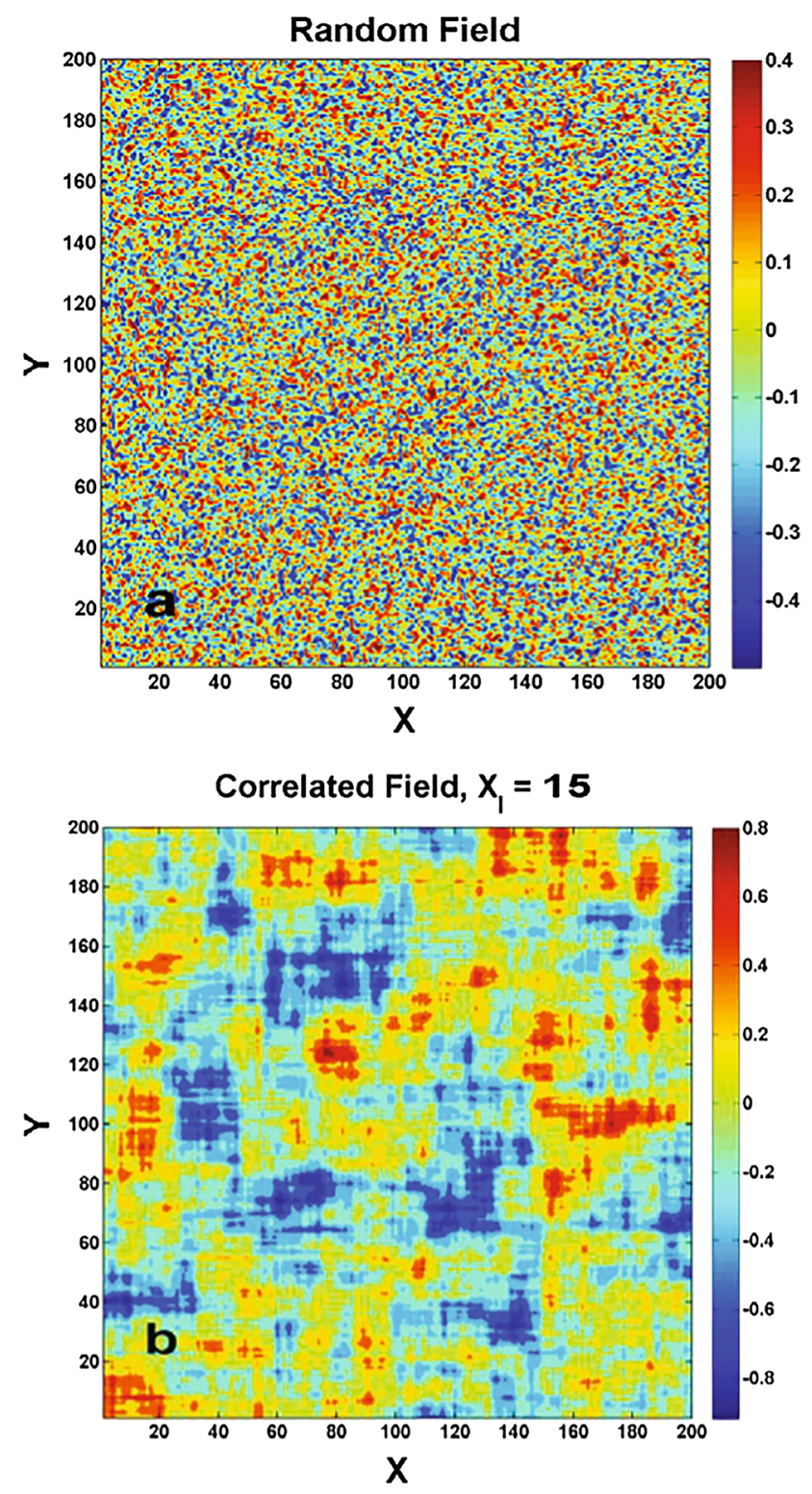

Fig. 1 a A field of random numbers and $\mathbf{b}$ the field in (a) after applying an exponentially decaying 2D correlation function. Structures are now apparent. In this work, we use this statistical framework and treat correlations as the statistical evidence for the presence of structures and vice versa

interpolation of raindrop rate followed by data analyses and a concluding discussion.

\section{Preliminary considerations}

Following the description in Jameson et al. (2015a), let us consider a random variable, $w$, as an element in a statistically homogeneous 2D field $W$. Let us also consider two instruments, one located at $\vec{r}$ and the other at $\vec{r}+\Delta \vec{r}$. Let us also assume that there are $M$ such pairs. At each instant in 
time, one can then define a correlation function between the $w$ observed over all $M$ pairs as

$\rho(\Delta \vec{r}, \theta)=\frac{\frac{1}{M} \sum_{i=1}^{M} w(\vec{r}, \theta)_{i} w(\vec{r}+\Delta \vec{r}, \theta)_{i}-\mu^{2}}{\sigma^{2}}$

where $\mu$ is the mean value of $w$ over $W$ and $\sigma^{2}$ is the variance over the interval. We have a time series of such correlations which we then average as denoted by the overbar, $\overline{\rho(\Delta \vec{r}, \theta)}$. Throughout the remainder of this work, the overbar is understood.

It is also worth noting that the ratio of the correlation function to the pair correlation function is just $\sigma^{2} / \mu^{2}$. [The pair correlation is the measurement of how, on average, the number of particles varies as a function of the spatial separation between two locations]. This is also the value of the pair correlation function at zero separation of measurement locations and is called the spatial pair correlation function coefficient (SPCFC). Thus, the correlation function can be converted to the pair correlation function after multiplying by the SPCFC (Jameson et al. 2015a).

There is more than one way to calculate $\rho(\Delta \vec{r}, \theta)$. One method is to perform a $2 \mathrm{D}$ correlation of the rectilinear $(x-$ $y$ ) matrix of values. This direct method is accomplished by shifting the matrix with respect to itself, multiplying the values in the overlapping area and the renormalizing to the values in over-lapping area for each shift in the $X$ - and $Y$ directions. Unlike the direct method, another approach is to use all the data simultaneously by calculating the 2D power spectrum of the spatial distribution of $w$. That is, one first calculates $\mu$ and removes it from all the elements in $W$. The Fourier transform of these $W$ times its complex conjugate is then the variance spectrum, i.e., the magnitude of the variance as a function of wavenumber (Blackman and Tukey 1975). If one then takes the inverse 2D Fourier transform of this variance spectrum, one derives the 2D correlation function by the Weiner-Khintchine theorem (Wiener 1930; Khintchine 1934). If $w$ were drop counts, for example, after normalization this correlation function is equivalent to $\rho(\Delta \vec{r}, \theta)$ or to the 2D pair correlation function after multiplying by the SPCFC. Calculations show that this method is less noisy than a direct matrix calculation. As for any sample by sample calculation of a correlation function even using the direct method, the data must be assumed or shown to be approximately statistically homogeneous. This is not always easy to demonstrate since the meteorology introduces correlations, although some methods exist (Anderson and Kostinski 2010). In earlier studies, these data have been shown to be essentially statistically homogeneous. However, because we will only be using temporal averages $\overline{\rho(\Delta \vec{r}, \theta)}$; this is not an issue because averages are calculated from summations which are commutative so that tends and correlations, which reflect order, do not matter. Correlations and trends can be readily destroyed by simply reshuffling of the data; yet the averages remain unaffected.

In addition, however, there is the so-called radial correlation function $\rho(\Delta r)$ which is just the azimuthal integration of $\rho(\Delta \vec{r}, \theta)$, i.e.

$\rho(\Delta r)=\int_{\theta} \rho(\Delta \vec{r}, \theta) \mathrm{d} \theta$

where $\theta$ is the azimuthal angle which, for this network, lies between 0 and $\pi / 2$ radians. In this work, $\rho(\Delta r)$ is calculated by converting the $2 \mathrm{D}$ correlation function in rectilinear coordinates into radius and azimuthal coordinates and then integrating at each $\Delta r$ over all the available angles. Multiplication by the SPCFC converts the radial correlation function into the so-called radial distribution function. Approximations to this function have been reported in the literature (e.g., Tapiador et al. 2010; Jaffrain and Berne 2012a) for the radar reflectivity factor and the rainfall rate, respectively.

\subsection{Data and analyses}

The network consists of three arms (Arm 1: detectors Q,R,S,T, U,V,W; Arm 2: H,J,K,N,P; Arm3: B,C,D,E,F,G), two being orthogonal (Arm1, Arm3) and the remainder (Arm2) bisecting the right angle. Detector A is at the origin. Instruments were then placed along each arm sequentially at $1.93,3.73,7.2,13.9,26.83,51.79$, and $100 \mathrm{~m}$ along each arm with the exception of Arm3 which was required to be less than $100 \mathrm{~m}$ because of restrictions imposed by being at an historical site. The layout is described in greater detail in and shown visually in Figs. A1-A3 in (Jameson et al. 2015a). The reader is referred to that source for further details except to note here that $1 \mathrm{~min}$ drop counts over 22 size bins are recorded every minute for all the instruments so that we can estimate the PSD and integrated quantities over all the detectors every minute.

\subsection{An example in intense, convective rain}

The rain event presented here occurred on 23 November 2013. While the event lasted $440 \mathrm{~min}$, the analyses presented in this work considers two periods, one from 15 to 52 min during intense convective rain having a network mean rainfall rate of $63 \mathrm{~mm} \mathrm{~h}^{-1}$. The other $310-400 \mathrm{~min}$ period during steadier, light rain having a network mean rainfall rate of $0.95 \mathrm{~mm} \mathrm{~h}^{-1}$ will be presented later. Earlier analyses for other purposes (Jameson et al. 2015a) using the technique of (Anderson and Kostinski 2010) revealed 
Fig. 2 Time-disdrometer plots of the data analyzed next. These show that all the variables exhibit continuity spatially throughout time so that spatial interpolation is a reasonable operation. The letters correspond to individual detectors plotted as a function of increasing distance from the origin of the network out to $100 \mathrm{~m}$. Furthermore, no detector appears to exhibit systematic error

these data to be statistically stationary in each of these two time periods.

We begin with a time-instrument plot of the intense, convective rain data for the variables to be analyzed below as illustrated in Fig. 2. There is apparent continuity in the spatial observations at different times so that the use of interpolation appears to be justified. Also note that there are no vertical temporal structures at any single instrument that would indicate the presence of over-counting or undercounting bias in any of the instruments.

Figure 3 is an example of the Watson interpolated rainfall rate for a $1 \mathrm{~min}$ period. The white circles with crosses indicate the locations of the optical disdrometers actually used in this work. (Note only 19 of the 21 instruments were operational during this period of early data collection.) In the $X$ - and diagonal directions, there are instruments out to $100 \mathrm{~m}$ from the origin of the network. In the $Y$-direction, the farthest instrument is a little over $71 \mathrm{~m}$ from the origin because of restrictions imposed at this historical preservation site. During this $1 \mathrm{~min}$, there were two locations with rainfall rates exceeding $220 \mathrm{~mm} \mathrm{~h}^{-1}$ which can happen at times in the moisture laden atmosphere in the southern United States.

In the convective rain, we first consider drops of $2.25 \mathrm{~mm}$ diameter. Each minute during the 38-min interval, $\rho(\Delta \vec{r}, \theta)$ is calculated beginning with the Watson interpolated fields of 1 min counts (equivalent to the concentration since the fall speed at one drop size is treated as constant) which extend $100 \mathrm{~m}$ in the $X$-direction and about $72 \mathrm{~m}$ in the $Y$-direction with interpolated values every meter. Coarser resolution (every $10 \mathrm{~m}$ ) did not produce any notable differences in the patterns except that the contours were more jagged. The 15-52 min mean value, $\mu$, is then subtracted from the $1 \mathrm{~min}$ interpolated values, and the Fourier technique is applied to derive the estimates of $\rho(\Delta \vec{r}, \theta)$. The 38 values of these interpolated fields are then averaged to produce the mean $2 \mathrm{D}$ correlation function which, when multiplied by the SPCFC, is also the mean 2D pair correlation function. To our knowledge, Fig. 4 is the first plot of such estimates of the 2D correlation functions over areas. The $X$ and $Y$ scales are identical even though their lengths are different. (Note that the separation lengths are necessarily less than $1 / 2$ of the geometric distances because of the symmetry of the correlation function over finite domains.). Obviously, the correlation function decreases along both $X$-and $Y$-directions. However, the most distinctive feature is that the contours do not form
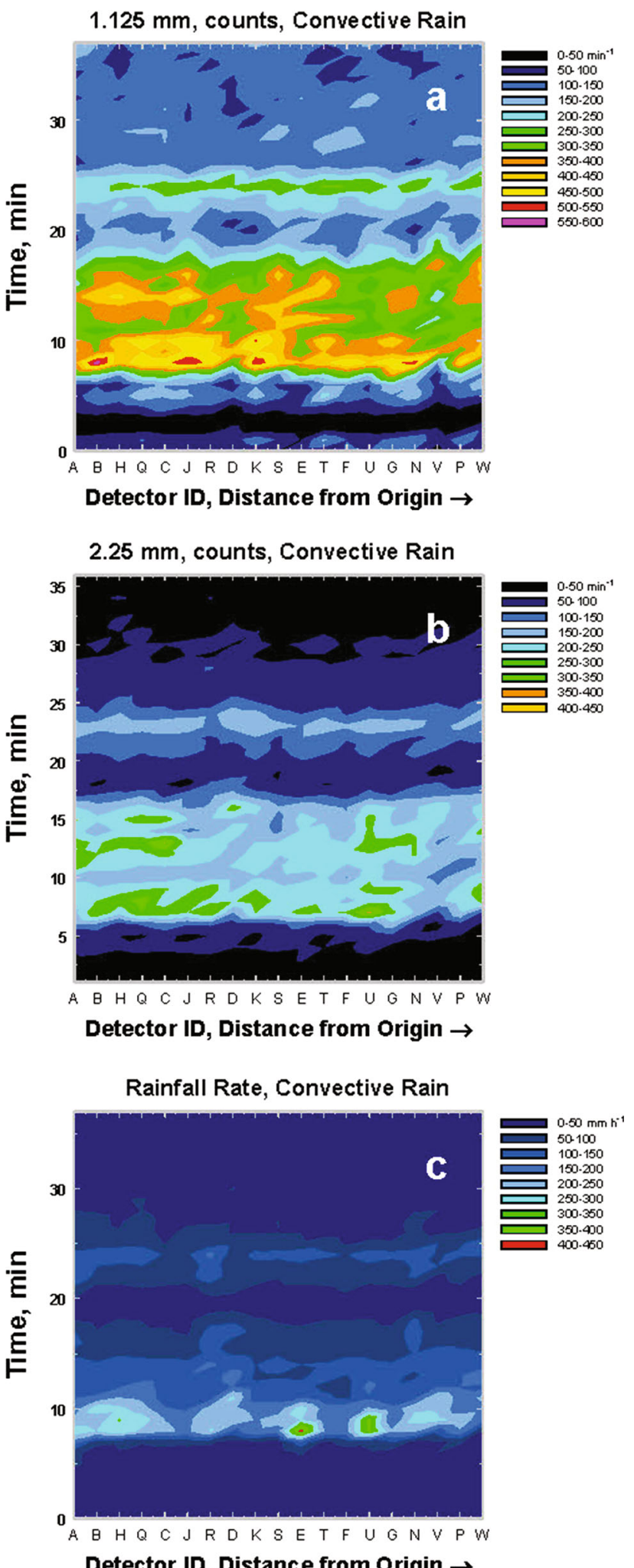

concentric arcs as one would expect for spatially symmetric functions.

Before commenting further on this observation, the radial correlation function, $\rho(\Delta r)$, is also computed (see 


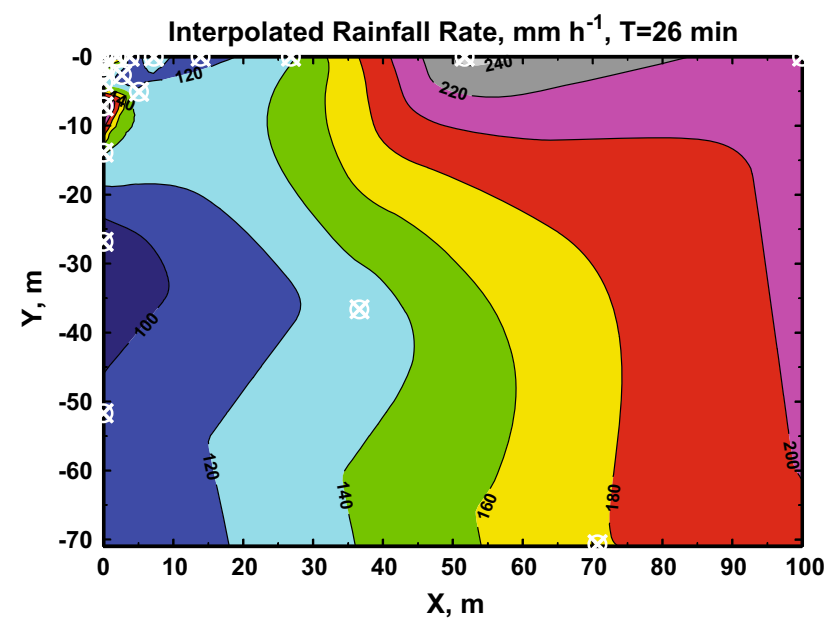

Fig. 3 An example of Watson interpolation during the convective rain event. The white circles with crosses denote the locations of the 19 disdrometers used in this study

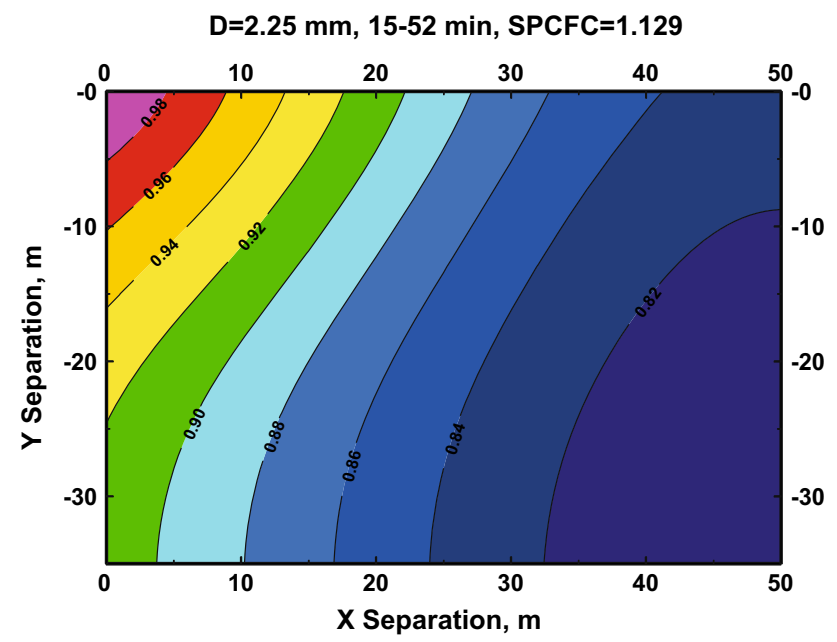

Fig. 4 The 15-52 min average spatial 2D correlation function in the convective rain for $2.25 \mathrm{~mm}$ sized drops. Multiplication by the spatial pair correlation function coefficient (SPCFC) yields the equivalent 2D pair correlation function. The asymmetry is noteworthy as discussed further in the text

above) and is plotted in Fig. 5a. The $x$ 's with error bars correspond to a limited number of values directly estimated and averaged over 1-120 min (Jameson et al. 2015a). Clearly, the observations are much more scattered in part because they were computed using a limited number of pairs of instruments but also because the pairs of instruments contributing to each separation are often at different angles with respect to each other. Consequently, spatial anisotropy likely contributes to the scatter as well. Furthermore, for the direct calculations, there are at most nine and sometimes only 2-3 pairs of instruments at any particular separation. By contrast, there are thousands of pairs
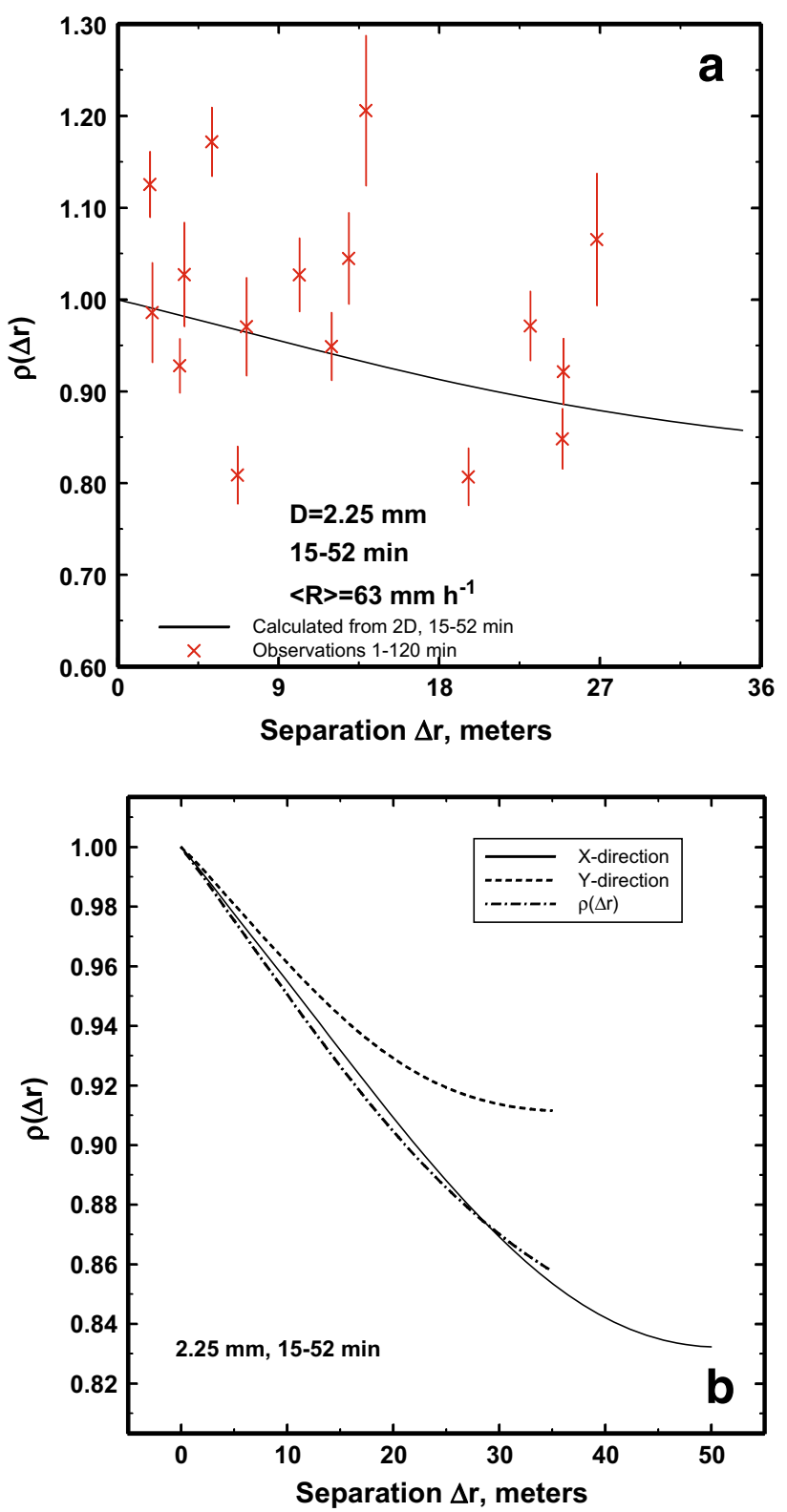

Fig. 5 a The radial correlation function, $\rho(\Delta r)$, calculated from the 2D spatial correlation function compared to a few limited direct measurements over a longer time period with $\pm 1 \sigma$ error bars (red) showing that $\rho(\Delta r)$ is reasonable. Observed values (red) exceeding unity are possible because of spatial anisotropy and other sampling effects as discussed in the text. b Is a comparison of $\rho(\Delta r)$ to the values of the $2 \mathrm{D}$ spatial correlation function along the two orthogonal axes of the network as discussed further in the text

at each separation when using the interpolated fields so that the results are much, much smoother. That is, the analysis approach using the interpolated fields likely provides a much more complete exploration of the correlation field by providing pairs over a wide range of angles and separations not available using only the direct observations. Furthermore, there are $2.5 \times 10^{7}$ such pairings as compared to the 
171 unique instrument pairs available for the direct calculations so that the accuracy is also much greater [see (6) in Jameson et al. (2015a) and the discussion in the summary]. Nevertheless, in spite of their obvious deficiencies, the observations are sufficient to provide a useful comparison with the analysis results. The observed values in Fig. 5a are derived by dividing the observed SPCFC by the interval average value. Thus, values in excess of unity can occur because of spatial anisotropy and local fluctuations in the SPCFC from the interval average value.

Returning again to the question of anisotropy of the $2 \mathrm{D}$ correlation function, the values of that function along both the $X$ - and $Y$-axes are plotted along with the radial correlation function in Fig. 5b. The anisotropy of the function between the two axes is now readily apparent. Moreover, because of the angular dependency of the radial correlation function, the more rapid decrease in the correlation function along the $X$ as opposed to the $Y$-direction drives $\rho(\Delta r)$ to more closely track what is happening in the $X$-direction. Moreover, because of its angular dependency, the radial correlation function is even slightly smaller than along the $X$-direction. Note, however, that the radial correlation function remains larger than 0.86 regardless of angle.

Next, we consider an entirely different set of measurements of drops about half the size as in the previous example, namely $D=1.125 \mathrm{~mm}$. Even though it is a completely different set of observations, Fig. 6 is quite similar to Fig. 4. However, Fig. 7a shows that $\rho(\Delta \vec{r}, \theta)$ decreases more rapidly with increasing separation than it did for $D=2.25 \mathrm{~mm}$, consistent with Fig. 7 in (Jameson et al. 2015a). Thus, the analysis technique apparently

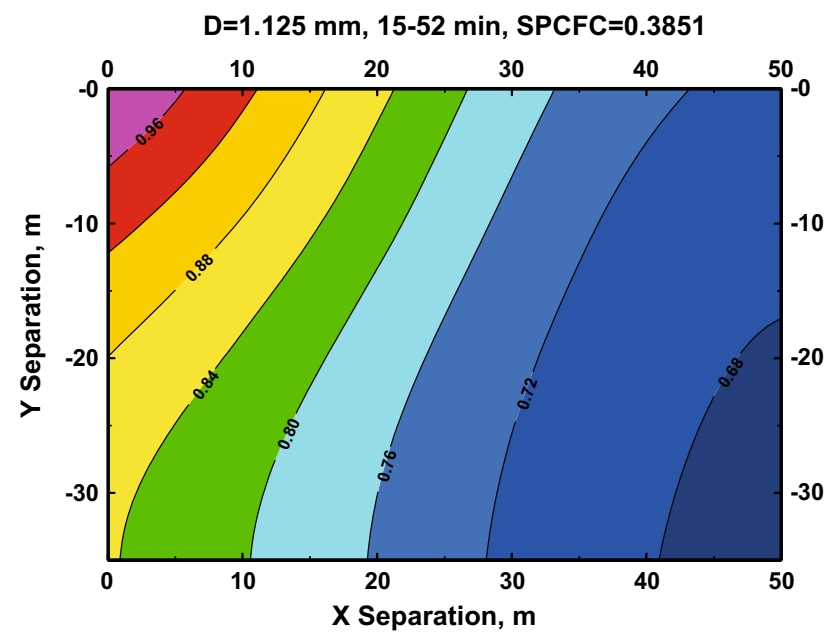

Fig. 6 The 15-52 min average spatial 2D correlation function in the convective rain for $1.125 \mathrm{~mm}$ sized drops. Multiplication by the spatial pair correlation function coefficient (SPCFC) yields the equivalent $2 \mathrm{D}$ pair correlation function. The asymmetry is apparent as discussed further in the text captures the essential differences between the two different drop sizes.

When the values along the axes are plotted, we find results also quite similar to those for the larger drop size as Fig. 7b illustrates. Hence, the anisotropy appears to be a feature of the $38 \mathrm{~min}$ of intense rain. Perhaps this is to be expected over such small spatial and temporal time scales in structured convective rain translating in a mean direction. To our knowledge, however, this is the first time this has been clearly demonstrated. Moreover, it appears to apply to the rainfall rate itself as shown next.
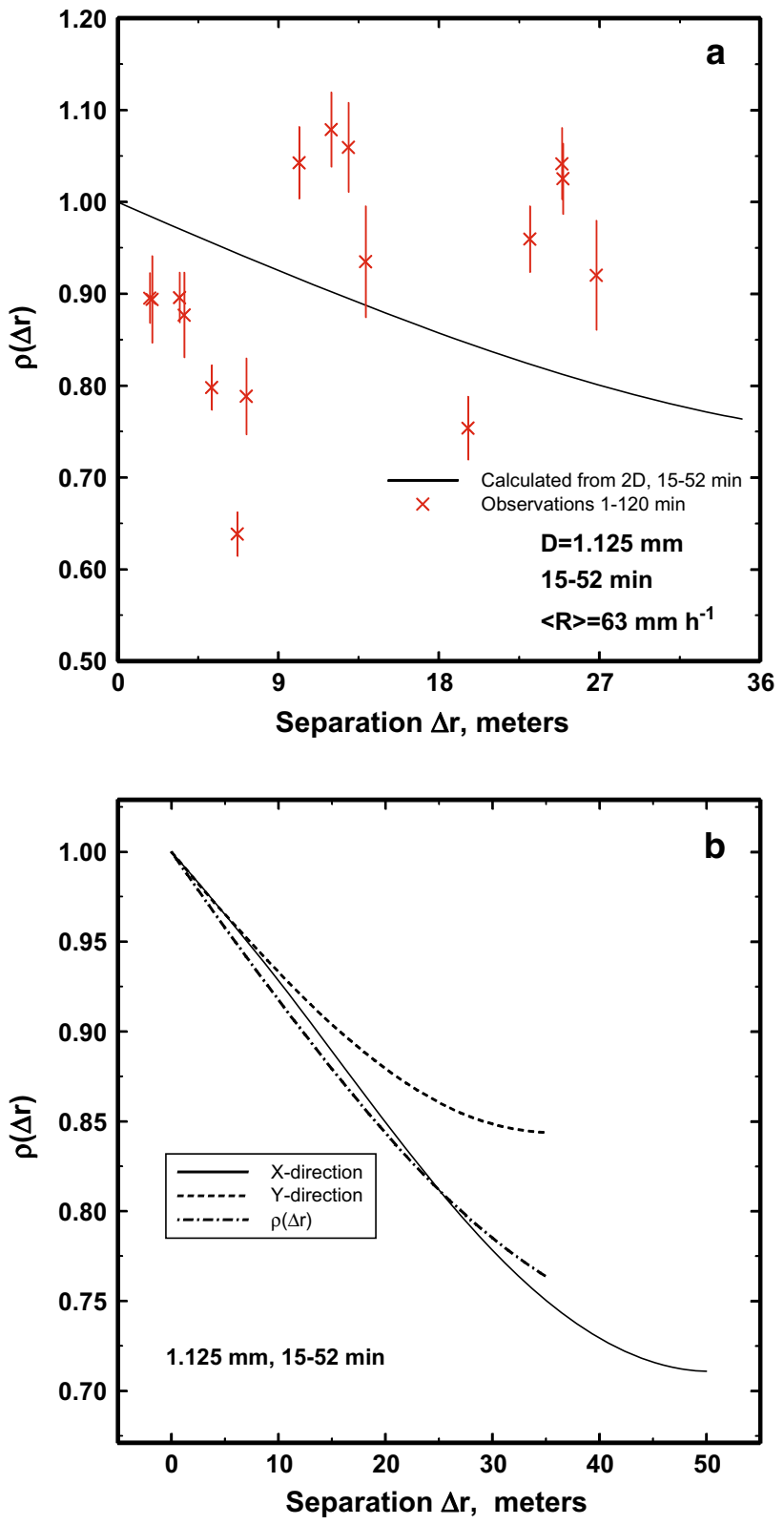

Fig. 7 As in Fig. 5 except for this smaller drop size illustrating the same kind of asymmetry as found for the larger drop size 
That is, for this set of measurements, even for quantities integrated overall drop sizes, the anisotropy appears in the rainfall rate itself as illustrated in Fig. 8 where once again the contours are not concentric arcs. However, the decrease in the correlation with increasing distance is more like that for the $2.25 \mathrm{~mm}$ drops than for the smaller drops. This is not surprising since it is the larger drops which are contributing most to the rainfall rate [see Fig. 4 in Jameson et al. (2015a)]. In these data, the radial correlation function for the rainfall rate $R$ (Fig. 9) most closely matches the values of $\rho(\Delta \vec{r}, \theta)$ along the $X$-axis, perhaps suggesting that precipitation elements are moving from the NW to the SE direction so that there is more persistent spatial correlation along the $Y$-axis since it is oriented along the NW-SE direction. Such a direction of motion is not uncommon. Of course, geometric asymmetries in the precipitation elements can yield the same result under the right circumstances.

Whatever the cause, for an exponentially decreasing radial correlation function for $R$, the rate of decrease of $\rho(\Delta r)$ would imply a correlation length of only about $239 \mathrm{~m}$ with complete decorrelation to the 0.01 level at $1.10 \mathrm{~km}$. This is considerably less than the $1.93 \mathrm{~km}$ decorrelation length found in Switzerland using a network of 16 optical disdrometers having a $1 \mathrm{~km}$ characteristic dimension (Jaffrain and Berne 2012a). This makes sense in no small part because with a $100 \mathrm{~m}$ network, an observer will not see all of the effects of the longer wavelengths (Jameson et al. 2015b). This suggests the importance of establishing a network of sparse instruments even up to a dimension of $20 \mathrm{~km}$, based upon a separate study of rainfall rate variability by Jameson and Larsen (2015). The technique presented here is well suited to an analysis of data from such an expanded sparse network of disdrometers, but at present, no such network currently exists that still retains these smaller scale structures while viewing larger scales as well.

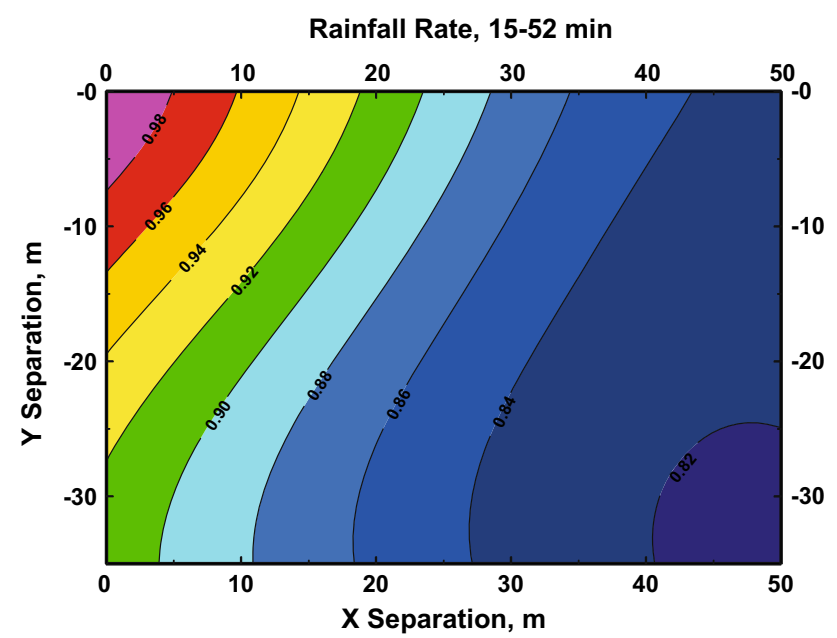

Fig. 8 Same as in Figs. 4 and 6 except for the rainfall rate. Again the asymmetry is apparent

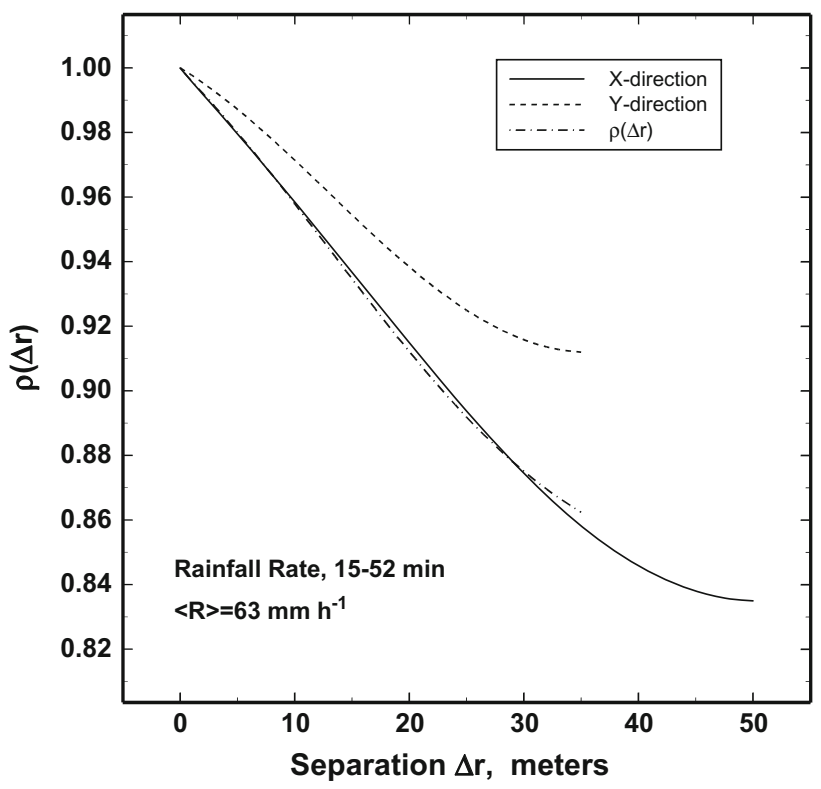

Fig. 9 A comparison of the radial correlation function for the rainfall rate to network axes values as in Figs. $5 \mathrm{~b}$ and $7 \mathrm{~b}$

\subsection{An example in light, steadier rain}

As one would expect, there is a considerable difference in the spatial correlation structure in lighter, steadier rain from that for the intense, convective rain. An abbreviated analysis of such an event is presented. The overall average rainfall rate was of $0.95 \mathrm{~mm} \mathrm{~h}^{-1}$. A plot of the data to be analyzed below (Fig. 10) shows spatial continuity at the different observation times; so that once again, interpolation appears to be reasonable.

When we consider $1.125 \mathrm{~mm}$ diameter drops, again there is asymmetry in the plot of $\rho(\Delta \vec{r}, \theta)$ (Fig. 11). It is also clear that the correlation decreases much more rapidly with increasing separation compared to the convective rain (Fig. 6). Another way of expressing this is that the lighter rain lacks a lot of the structure (which is what is meant by coherence) that appears in the more convective rain. Indeed the radial correlation function (Fig. 12) decreases to 0.55 in the same distance it took to diminish to about 0.86 in the convective rain. This more rapid decorrelation is consistent with the observations reported in Jameson et al. (2015a). Also in contrast to the convective rain, the correlation along the $X$-axis is larger than along the $Y$-axis out to about $30 \mathrm{~m}$. Moreover, the radial correlation $\rho(\Delta r)$ is smaller than it is along either the $X$ or $Y$-axis because of the angular dependence of $\rho(\Delta \vec{r}, \theta)$. That is, the anisotropy of the correlation function apparently plays a bigger role in this lighter rain than in the convective rain perhaps because of the reduced spatial correlation.

Finally, when we consider the rainfall rate, this anisotropy is even more apparent in both Figs. 13 and 14. The 

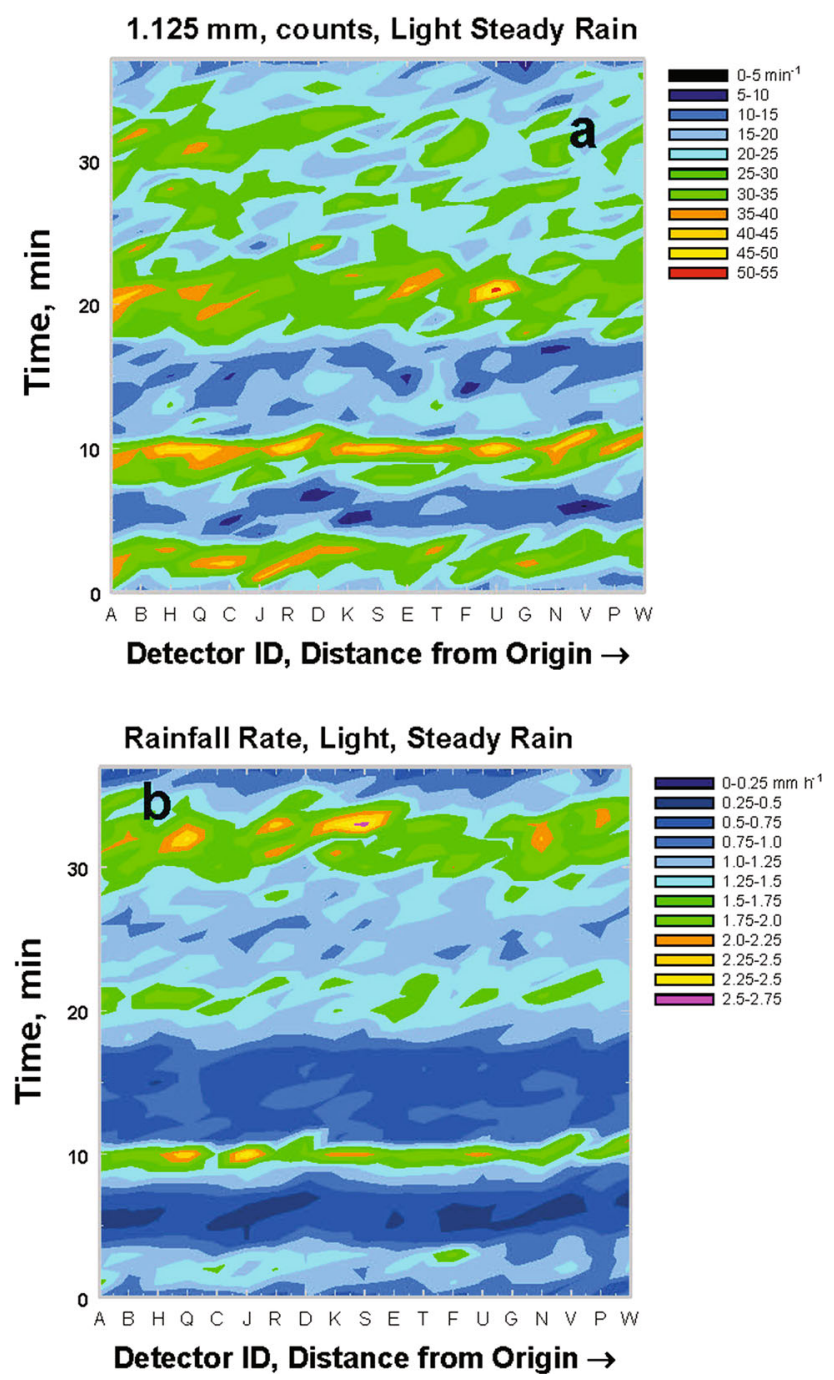

Fig. 10 Time-disdrometer plots of the steady, light rain data. These show that all the variables exhibit continuity spatially through time so that spatial interpolation is a reasonable operation

decrease in the radial correlation function for the rainfall rate is much stronger in the lighter rain compared to the more intense convective rain as illustrated in Fig. 15. $\rho(\Delta \mathrm{r})$ decreases to about 0.68 at $36 \mathrm{~m}$ implying that for an exponentially decreasing correlation function, the correlation length is only about $93 \mathrm{~m}$ with complete decorrelation to the 0.01 level at only $428 \mathrm{~m}$. This is smaller than $1 / 3$ of those values in the convective rain example. While one may intuitively expect more wide-spread light rain to be more uniform and, therefore, more correlated from place to place, the opposite is true so that in a real sense, the lighter rain is actually more spatially random than is the convective rain, but the variability is just not as apparent. However, more studies are required to explore the potential generality of the results from these two examples.

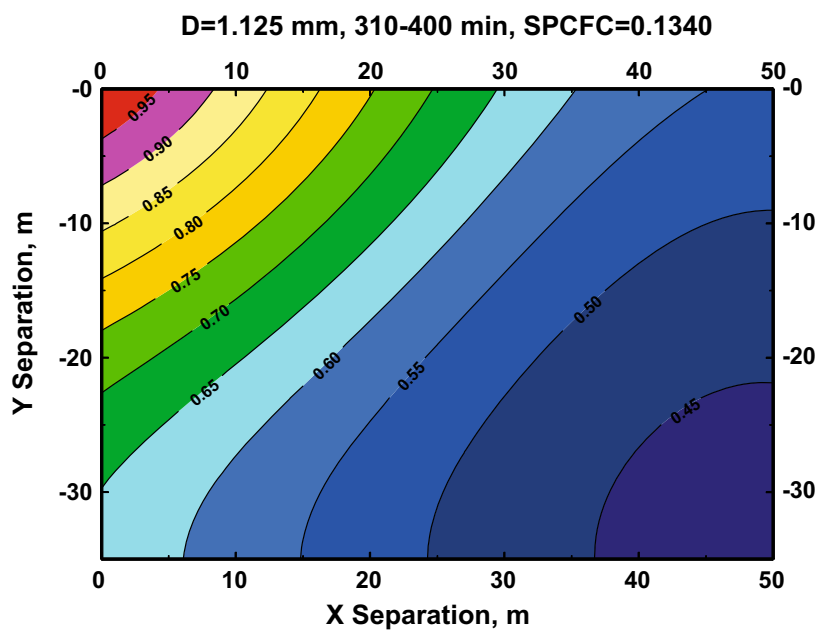

Fig. 11 Similar to Fig. 7 except for the steady, light rain. Note the more rapid decorrelation than in the convective rain. Asymmetry is still present at lower correlations, but it differs from that in the convective rain as discussed in the text

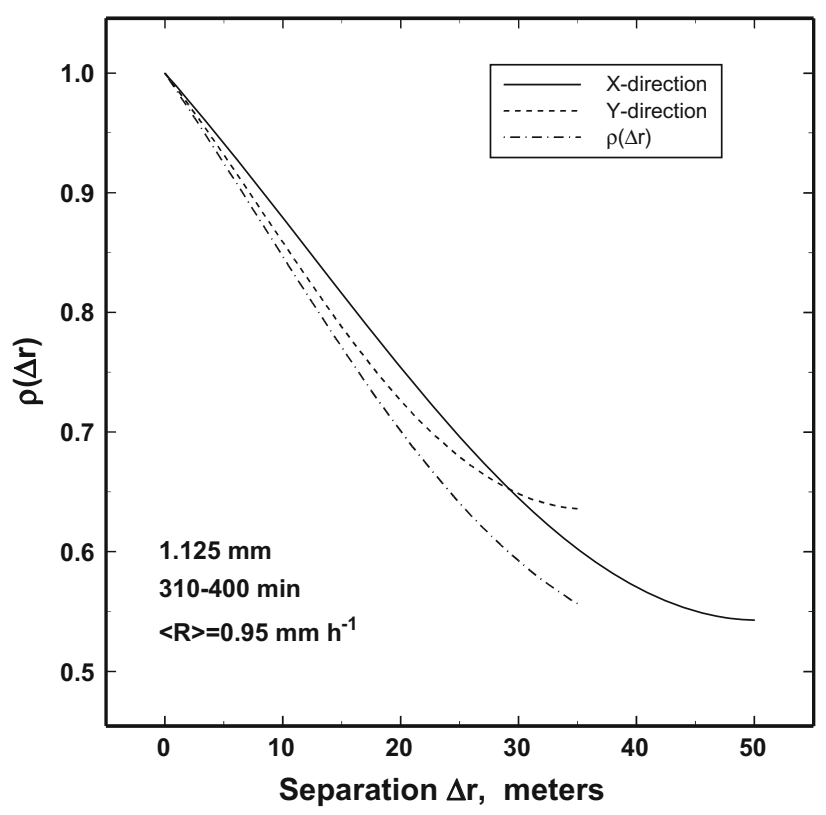

Fig. 12 A comparison of the radial correlation function to values of the $2 \mathrm{D}$ correlation function along the network axes. Unlike in the convective rain, the values along the $X$-axis exceed those along the $Y$ axis during this time period

\section{Summary}

In this brief note, an approach for deriving the 2D correlation function over a sparse network of optical disdrometers is developed. For the first time, we get a glimpse of just what such functions might look like over a small domain of $71 \mathrm{~m} \times 100 \mathrm{~m}$ defined by 19 optical disdrometers. Such a small network can only yield 171 spatial pairs 


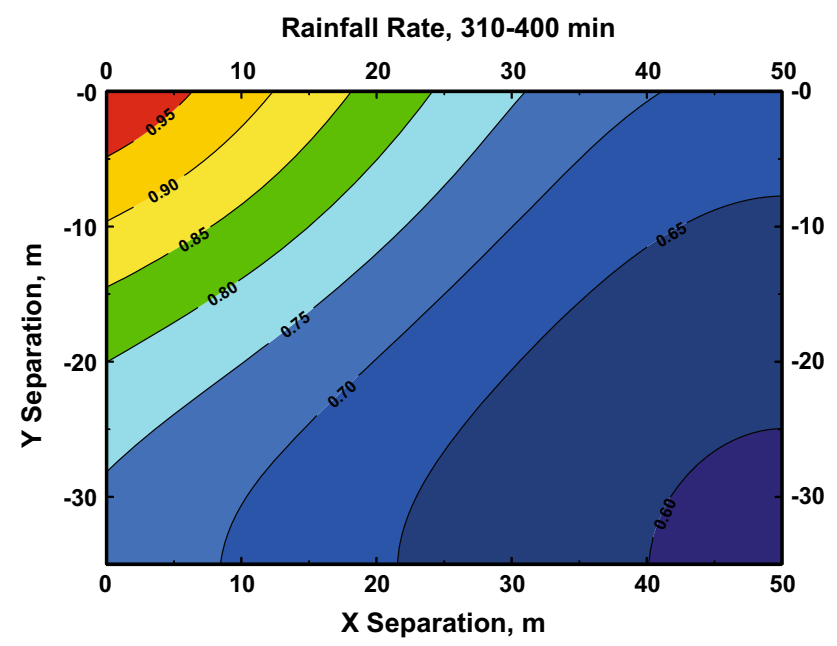

Fig. 13 A plot of the 2D correlation function for the rainfall rate during the steadier, lighter rain. The decorrelation is spatially faster than for the convective rain and asymmetry is again evident

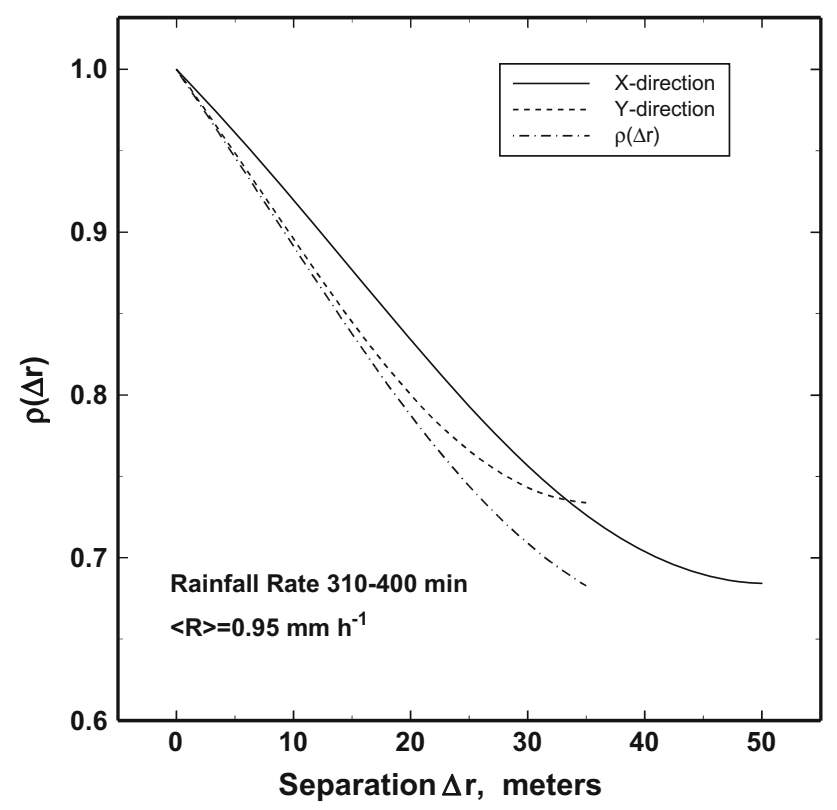

Fig. 14 A comparison of the radial correlation function to values of the 2D correlation function along the network axes for the rainfall rate. Unlike in the convective rain, the values along the $X$-axis exceed those along the $Y$-axis during this time period

which are much too limited to define any 2D structure over an area. However, by using a conservative interpolation scheme that remains true to the data and yet does not introduce artificial features so that the natural correlation function remains unaltered, the spatial pairs are increased to approximately $2.5 \times 10^{7}$ pairs with a data value every meter in both directions every minute. In part because it is impractical to compute each pair directly but for other reasons as well, these interpolated fields are converted to

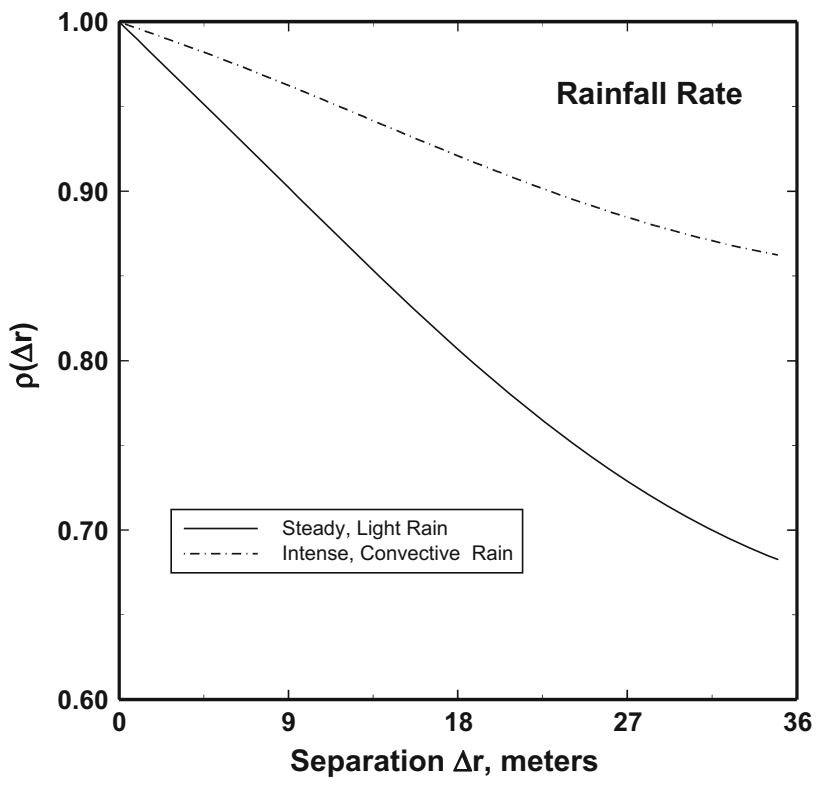

Fig. 15 A comparison of the radial correlation functions in the two types of rain

fields of zero mean numbers. The Fourier transform of this field times its complex conjugate then yields the field of variances which can be transformed into the $2 \mathrm{D}$ spatial correlation function using an inverse Fourier transform (via the Wiener-Khintchine theorem). With a set of $911 \mathrm{~min}$ interpolated fields using a $1 \mathrm{~m}$ resolution over a $71 \mathrm{~m} \times 100 \mathrm{~m}$ grid, for example, these calculations take under $1 \mathrm{~s}$ on a PC.

The uncertainty in the estimation of the pair correlation function is given by Eq. 6 in Jameson et al. (2015a). It does not depend much at all on the number of counts as it does on the inverse square root of the number of pairs of detectors $(1 / \sqrt{ } M)$ multiplied by the inverse square root of the number of $1 \mathrm{~min}$ observations $(1 / \sqrt{ } \mathcal{N})$ with a very small contribution going as the inverse of the mean number of counts, $\mu$. This latter term is negligible even for light rain when the mean counts per minute were at least 100 . We considered two types of rain: one, a 38-min intense convective rain and the other, a light, steady rain lasting $91 \mathrm{~min}$. Hence, for the more intense rain and for the lighter rain the $1 / \sqrt{ } \mathcal{N}$ terms are 0.16 and 0.10 , respectively. In both cases, the pairs of points for each separation is on the order of 252,000 so that the relative errors in the estimates of $\overline{\rho(\Delta \vec{r}, \theta)}$ are on the order of $0.03 \%$ in the heavy rain and $0.02 \%$ in the light rain. Thus, the increased variability in the light rain compared to the heavier rain appears to be real. Obviously, however, there are only two examples so the results should not be treated with generality.

In each case, the 2D spatial correlation function was computed for each minute as just outlined. These were then averaged over the entire interval to produce the mean $2 \mathrm{D}$ 
spatial correlation function for each type of rain and for select drop sizes as well as for the rainfall rates.

The results showed that in all cases, the $2 \mathrm{D}$ spatial correlation function was anisotropic. The radial correlation function is derived by integrating the $2 \mathrm{D}$ correlation function in the azimuthal direction at each radial distance from the origin. Plots of the radial correlation function also confirm this asymmetry. While not consistent with the isotropy assumed by Jaffrain and Berne (2012a), it is consistent with some of the findings by Tapiador et al. (2010) over distances of several hundreds of meters to kilometers at least for the radar reflectivity factor.

To rule out some unknown factors including any unknown potential effects of interpolation, an independent check is provided using results from a numerical experiment. In Jameson (2015) measurements by a single JossWaldvogel disdrometer were used to construct $2 \mathrm{D}$ rain fields using the observed concentration distributions as well as the observed temporal correlation functions at each drop size. The results for each drop size were then combined to produce patterns of the rainfall rate and other variables. One example for the rainfall rate is provided in Fig. 16a. [Note that the apparent 'boxy look' arises from resolution limitations and is not a processing artifact as explained further in Jameson (2015)]. When the 2D correlation function is computed for Fig. 16a using the Fourier method given above, Fig. 16b shows that there is also significant asymmetry. For example, the 0.3 contour line intersects the $Y$-axis at $2 \mathrm{~km}$ but intersects it at $3.4 \mathrm{~km}$ at the $X$-axis. For completeness, the calculated radial correlation function is given in Fig. 17 where it appears to be best represented by an exponential function. These results are independent of any interpolation. Thus, we conclude that the results presented in this work are likely valid and that anisotropy may exist at times at least over shorter intervals and smaller areas. This is important because it reflects anisotropy in physical processes producing the precipitation at the ground. Again, however, these results are derived using a limited number of cases so that any generalization requires more research beyond the scope of this method paper.

As intriguing as these findings are, however, they are far from complete. Observations like these are required over many more cases and over larger domains and over longer times, but at least the approach outlined here is readily scalable. Over long time periods, other conditions, and longer length scales, such as anisotropy may disappear on average. However, since there is nothing special about these data, the results suggest that for some rain events of reasonable duration, one might reasonably expect some degree of anisotropy. Moreover, even a few examples of this anisotropy presented here suggests that one cannot simply follow a drop in time and height and expect to explain all of the processes affecting the evolution of that
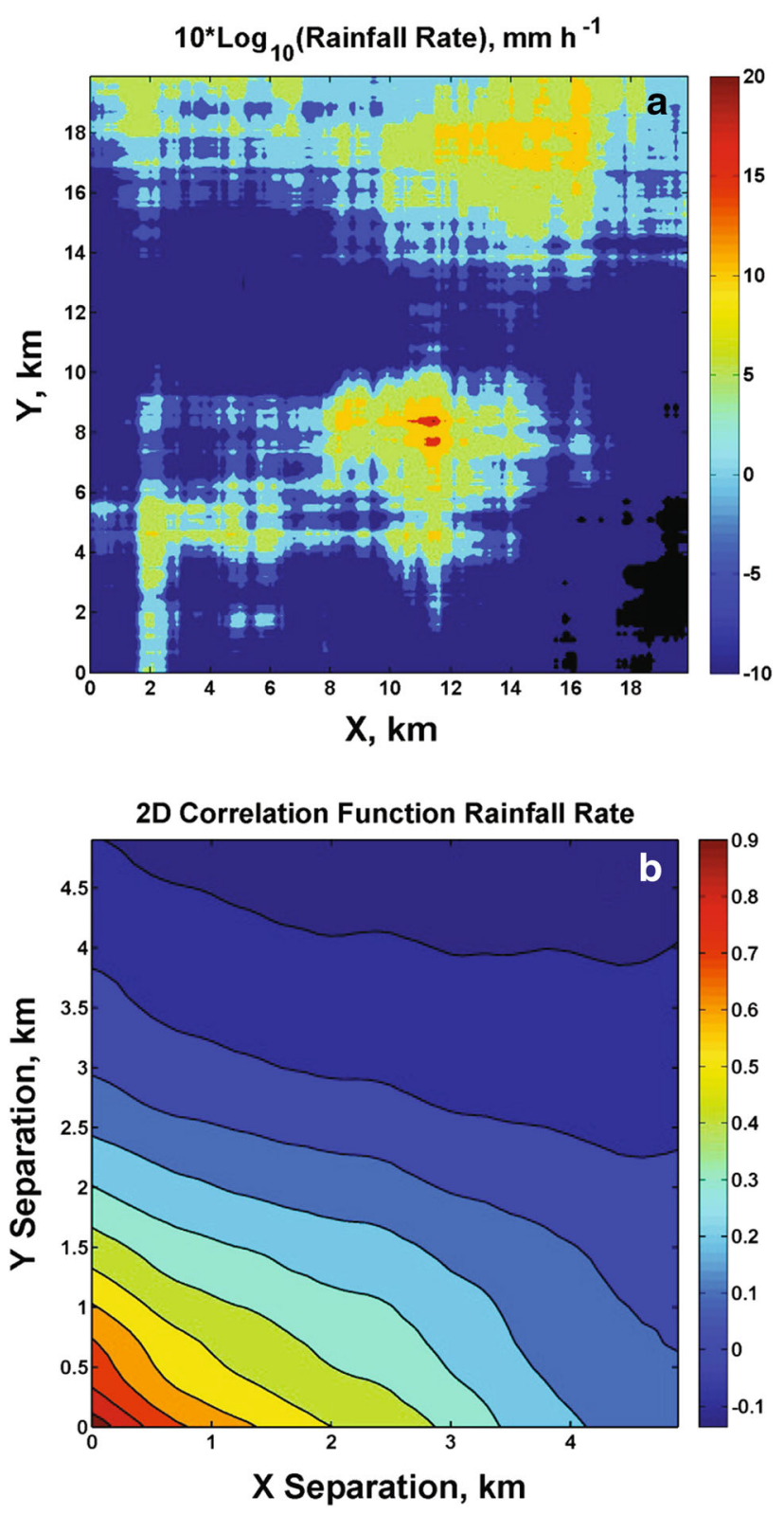

Fig. 16 a The rainfall rate from a realization using data from a single disdrometer as described in the text and in greater detail in (Jameson 2015). b The 2D spatial correlation function corresponding to (a). This approach involves no interpolation, but again the 2D spatial correlation function exhibits significant anisotropy

drop without considering all of the geometric dimensions. Hence, a full understanding of the microphysical evolution of rain using numerical simulations will require the inclusion of this multi-dimensionality.

Perhaps most importantly, however, this work provides a method for using the inevitable sparse network of disdrometers to provide information about the 2D correlation function which is, for all practical purposes, difficult if not impossible to determine otherwise. Such information is important to the development of a better understanding of 


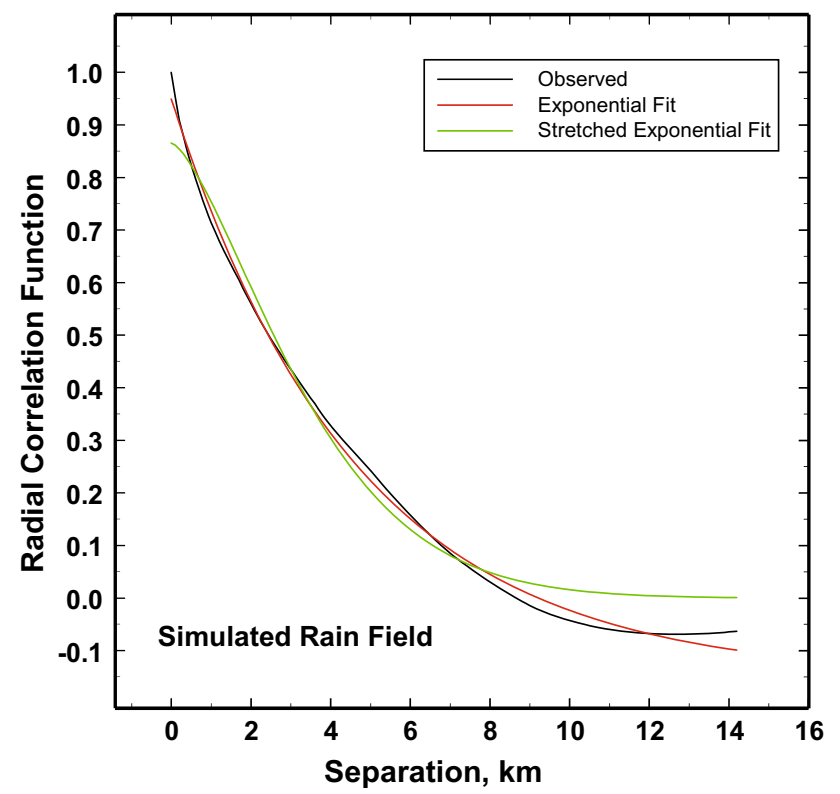

Fig. 17 For completeness, the radial correlation function corresponding to Fig. 16 is plotted and is well fit by an exponential function

soil erosion, a multi-billion a year loss to agriculture worldwide (Pimentel et al. 1995; Uri and Lewis 1999). Ignoring the vertical dimension, soil has two-dimensional structures which are then convolved with the two-dimensional structures of the rain during erosion. This is at the cutting edge of research into erosion which is by its nature a fine scale process.

Acknowledgments This work was supported for AJ by the National Science Foundation (NSF) under grants AGS130087 and AGS1532423 as well as by the United States Social Security Administration. Support for ML came from the National Science Foundation under grants AGS1230240 and AGS1532977. The authors are also especially grateful to the students of Prof. Larsen, specifically to Joerael Harris, Robert Lemasters, Katelyn O'Dell, Joshua Teves and Michael Chute whose diligent efforts made this network a functioning reality.

Open Access This article is distributed under the terms of the Creative Commons Attribution 4.0 International License (http://crea tivecommons.org/licenses/by/4.0/), which permits unrestricted use, distribution, and reproduction in any medium, provided you give appropriate credit to the original author(s) and the source, provide a link to the Creative Commons license, and indicate if changes were made.

\section{References}

Ahrens B, Beck A (2008) On upscaling of rain-gauge data for evaluating numerical weather forecasts. Meteorol Atmos Phys 99:155-167. doi:10.1007/s00703-007-0261-8

Anderson A, Kostinski A (2010) Reversible record breaking and variability: temperature distributions across the globe. J Appl Meteorol Climatol 49:1681-1691. doi:10.1175/2010JAMC2407.1
Best AC (1950) The size distribution of raindrops. Q J R Meteorol Soc 76:16-36. doi:10.1002/qj.49707632704

Blackman RB, Tukey JW (1975) The measurement of power spectra. From the point of view of communications engineering. Dover Publ, New York

Caracciolo C, Napoli M, Porcù F et al (2012) Raindrop size distribution and soil erosion. J Irrig Drain Eng 138:461-469. doi:10.1061/(ASCE)IR.1943-4774.0000412

Crane RK (1990) Space-time structure of rain rate fields. J Geophys Res Atmos 95:2011-2020. doi:10.1029/JD095iD03p02011

Droegemeier KK, Smith JD, Businger S et al (2000) Hydrological aspects of weather prediction and flood warnings: report of the Ninth Prospectus Development Team of the U.S. Weather Research Program. Bull Am Meteorol Soc 81:2665-2680. doi:10.1175/1520-0477(2000)081<2665:HAOWPA > 2.3.CO;2

Ferraris L, Rudari R, Siccardi F (2002) The uncertainty in the prediction of flash floods in the northern Mediterranean environment. J Hydrometeorol 3:714-727. doi:10.1175/15257541(2002)003<0714:TUITPO > 2.0.CO;2

Hodges DD, Watson RJ, Paulson KS (2005) Techniques for the spatial downscaling of rain for application in SHF propagation modelling. IEEE, pp 55-58

Huebener H, Born K, Kerschgens M (2007) Downscaling heavy rainfall in the subtropics - a simple approach for dynamical nesting. Adv Geosci 10:9-16

Jaffrain J, Berne A (2012a) Quantification of the small-scale spatial structure of the raindrop size distribution from a network of disdrometers. J Appl Meteorol Climatol 51:941-953. doi:10. 1175/JAMC-D-11-0136.1

Jaffrain J, Berne A (2012b) Influence of the subgrid variability of the raindrop size distribution on radar rainfall estimators. J Appl Meteorol Climatol 51:780-785. doi:10.1175/JAMC-D-11-0185. 1

Jameson AR (2015) A Bayesian method for upsizing single disdrometer drop size counts for rain physics studies and areal applications. IEEE Trans Geosci Remote Sens 53:335-343. doi:10.1109/TGRS.2014.2322092

Jameson AR, Kostinski AB (1999) Fluctuation properties of precipitation. Part V: distribution of rain rates - theory and observations in clustered rain. J Atmos Sci 56:3920-3932. doi:10.1175/ 1520-0469(1999)056<3920:FPOPPV > 2.0.CO;2

Jameson AR, Kostinski AB (2000) Fluctuation properties of precipitation. Part VI: observations of hyperfine clustering and drop size distribution structures in three-dimensional rain. J Atmos Sci 57:373-388. doi:10.1175/1520-0469(2000)057<0373: FPOPPV $>2.0 . \mathrm{CO} ; 2$

Jameson AR, Kostinski AB (2010a) Partially coherent backscatter in radar observations of precipitation. J Atmos Sci 67:1928-1946. doi:10.1175/2010JAS3336.1

Jameson AR, Kostinski AB (2010b) Direct observations of coherent backscatter of radar waves in precipitation. J Atmos Sci 67:3000-3005. doi:10.1175/2010JAS3488.1

Jameson AR, Larsen ML (2015) The variability of the rainfall rate as a function of area: variability of rainfall rates over areas. J Geophys Res Atmos. doi:10.1002/2015JD024126

Jameson AR, Larsen ML, Kostinski AB (2015a) Disdrometer network observations of finescale spatial-temporal clustering in rain. J Atmos Sci 72:1648-1666. doi:10.1175/JAS-D-14-0136.1

Jameson AR, Larsen ML, Kostinski AB (2015b) On the variability of drop size distributions over areas. J Atmos Sci 72:1386-1397. doi:10.1175/JAS-D-14-0258.1

Johnson GE (1994) Constructions of particular random processes. Proc IEEE 82:270-285. doi:10.1109/5.265353

Khintchine A (1934) Korrelationstheorie der stationaren stochastischen Prozesse. Math Ann 109:604-615. doi:10.1007/ BF01449156 
Kinnell PIA (2005) Raindrop-impact-induced erosion processes and prediction: a review. Hydrol Process 19:2815-2844. doi:10. 1002/hyp.5788

Kostinski AB, Jameson AR (1997) Fluctuation properties of precipitation. Part I: on deviations of single-size drop counts from the Poisson distribution. J Atmos Sci 54:2174-2186. doi:10.1175/ 1520-0469(1997)054<2174:FPOPPI >2.0.CO;2

Kostinski AB, Jameson AR (2000) On the spatial distribution of cloud particles. J Atmos Sci 57:901-915. doi:10.1175/15200469(2000)057<0901:OTSDOC>2.0.CO;2

Kostinski AB, Shaw RA (2001) Scale-dependent droplet clustering in turbulent clouds. J Fluid Mech 434:389-398

Kostinski AB, Shaw RA (2005) Fluctuations and luck in droplet growth by coalescence. Bull Am Meteorol Soc 86:235-244. doi:10.1175/BAMS-86-2-235

Kostinski AB, Larsen ML, Jameson AR (2006) The texture of rain: exploring stochastic micro-structure at small scales. Meas Parameter Rainfall Microstruct 328:38-45. doi:10.1016/j.jhy drol.2005.11.035

Lanza LG, Ramirez JA, Todini E (2001) Stochastic rainfall interpolation and downscaling. Hydrol Earth Syst Sci 5:139-143

Lee GW, Zawadzki I (2005) Variability of drop size distributions: noise and noise filtering in disdrometric data. J Appl Meteorol 44:634-652. doi:10.1175/JAM2222.1

Lee CK, Lee GW, Zawadzki I, Kim K-E (2009) A preliminary analysis of spatial variability of raindrop size distributions during stratiform rain events. J Appl Meteorol Climatol 48:270-283. doi:10.1175/2008JAMC1877.1

Mannshardt-Shamseldin EC, Smith RL, Sain SR et al (2010) Downscaling extremes: a comparison of extreme value distributions in point-source and gridded precipitation data. Ann Appl Stat 4:484-502. doi:10.1214/09-AOAS287

Marshall JS, Palmer WMK (1948) The distribution of raindrops with size. J Meteorol 5:165-166. doi:10.1175/15200469(1948)005<0165:TDORWS $>2.0 . C O ; 2$

Meusburger K, Steel A, Panagos P et al (2012) Spatial and temporal variability of rainfall erosivity factor for Switzerland. Hydrol Earth Syst Sci 16:167-177. doi:10.5194/hess-16-167-2012

Pimentel D, Harvey C, Resosudarmo P et al (1995) Environmental and economic costs of soil erosion and conservation benefits. Science 267:1117-1123. doi:10.1126/science.267.5201.1117

Rebora N, Ferraris L, von Hardenberg J, Provenzale A (2006) Rainfall downscaling and flood forecasting: a case study in the Mediterranean area. Nat Hazards Earth Syst Sci 6:611-619

Sangati M, Borga M (2009) Influence of rainfall spatial resolution on flash flood modelling. Nat Hazards Earth Syst Sci 9:575-584. doi: 10.5194/nhess-9-575-2009

Sassi MG, Leijnse H, Uijlenhoet R (2014) Sensitivity of power functions to aggregation: bias and uncertainty in radar rainfall retrieval. Water Resour Res 50:8050-8065. doi:10.1002/ 2013WR015109

Shaw RA, Reade WC, Collins LR, Verlinde J (1998) Preferential concentration of cloud droplets by turbulence: effects on the early evolution of cumulus cloud droplet spectra. J Atmos Sci 55:1965-1976. doi:10.1175/1520-0469(1998)055<1965: PCOCDB $>2.0 . \mathrm{CO} ; 2$

Shaw RA, Kostinski AB, Larsen ML (2002) Towards quantifying droplet clustering in clouds. Q J R Meteorol Soc 128:1043-1057. doi:10.1256/003590002320373193

Sibson (1981) A brief description of natural neighbor interpolation. In: Barnett (ed) Interpreting multivariate data. Wiley, New York, pp 21-36

Steiner M, Smith JA, Uijlenhoet R (2004) A microphysical interpretation of radar reflectivity-rain rate relationships. J Atmos Sci 61:1114-1131

Tapiador FJ, Checa R, de Castro M (2010) An experiment to measure the spatial variability of rain drop size distribution using sixteen laser disdrometers: spatial variability of the rain DSD. Geophys Res Lett. doi:10.1029/2010GL044120

Tokay A, Bashor PG (2010) An experimental study of small-scale variability of raindrop size distribution. J Appl Meteorol Climatol 49:2348-2365. doi:10.1175/2010JAMC2269.1

Tustison B, Harris D, Foufoula-Georgiou E (2001) Scale issues in verification of precipitation forecasts. J Geophys Res Atmos 106:11775-11784. doi:10.1029/2001JD900066

Uijlenhoet R, Smith JA, Steiner M (2003a) The microphysical structure of extreme precipitation as inferred from ground-based raindrop spectra. J Atmos Sci 60:1220-1238. doi:10.1175/15200469(2003)60<1220:TMSOEP $>2.0 . C O ; 2$

Uijlenhoet R, Steiner M, Smith JA (2003b) Variability of raindrop size distributions in a squall line and implications for radar rainfall estimation. J Hydrometeorol 4:43-61. doi:10.1175/15257541(2003)004<0043:VORSDI >2.0.CO;2

Uri ND, Lewis JA (1999) Agriculture and the dynamics of soil erosion in the United States. J Sustain Agric 14:63-82. doi:10. 1300/J064v14n02_07

van de Beek CZ, Leijnse H, Torfs PJJF, Uijlenhoet R (2012) Seasonal semi-variance of Dutch rainfall at hourly to daily scales. Adv Water Resour 45:76-85. doi:10.1016/j.advwatres.2012.03.023

Venugopal V, Foufoula-Georgiou E, Sapozhnikov V (1999a) A space-time downscaling model for rainfall. J Geophys Res 104:19705. doi:10.1029/1999JD900338

Venugopal V, Foufoula-Georgiou E, Sapozhnikov V (1999b) Evidence of dynamic scaling in space-time rainfall. J Geophys Res 104:31599. doi:10.1029/1999JD900437

Verlinde J (2011) In the Pangani Basin in Tanzania. Masters Thesis, Delft University of Technology

Watson DF (1992) Contouring: a guide to the analysis and display of spatial data: with programs on diskette, 1st edn. Pergamon Press, Oxford

Wexler R (1948) Rain intensities by radar. J Meteorol 5:171-173. doi:10.1175/1520-0469(1948)005<0171:RIBR >2.0.CO;2

Wiener N (1930) Generalized harmonic analysis. Acta Math 55:117-258. doi:10.1007/BF02546511 